\title{
Planejamento otimizado da produção e logística de empresas produtoras de sementes de milho: um estudo de caso
}

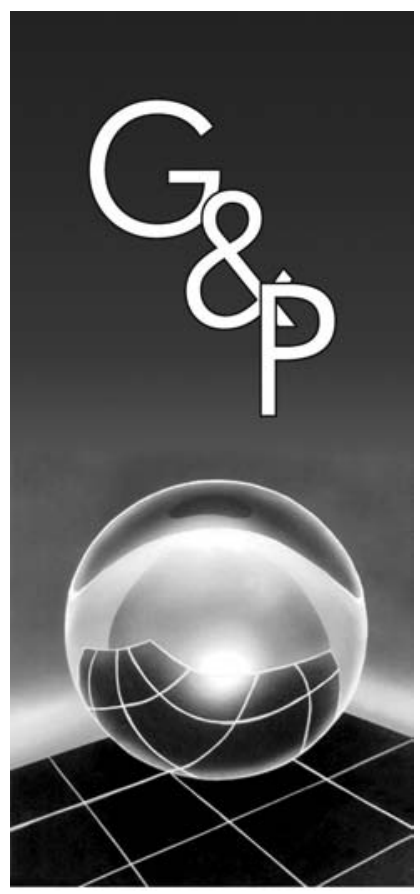

\author{
Rogério de Ávila Ribeiro Junqueira \\ Reinaldo Morabito
}

\begin{abstract}
Resumo
Em um trabalho anterior (JUNQUEIRA; MORABITO, 2006) foi apresentado um modelo de otimização linear para auxiliar nas decisões do planejamento tático da produção, estocagem e transporte de sementes de milho, de forma a minimizar custos de produção, logísticos e fiscais, e satisfazer restrições de programação da colheita, capacidade das plantas e demanda dos clientes. $O$ modelo foi testado apenas em situações simplificadas da realidade. No presente trabalho, analisamos a aplicação deste modelo para gerar um planejamento agregado otimizado em um estudo de caso de uma safra completa de uma empresa do setor de sementes de milho. Foram gerados e avaliados diversos cenários por meio deste modelo, considerando, entre outros, os custos fiscais de ICMS e de secagem de sementes, e os resultados obtidos foram comparados com os do método de planejamento utilizado pela empresa. Estes resultados mostram que reduções de custo importantes podem ser obtidas nos planos de produção e logística, quando consideradas as premissas presentes no modelo.
\end{abstract}

Palavras-chave: Sementes de milho. Planejamento agregado da produção e logística. Otimização linear. Planejamento tributário. ICMS.

\section{Introdução}

Abordagens de pesquisa operacional têm sido utilizadas para apoiar decisões agregadas de produção, estocagem e transporte em diversos sistemas de planejamento da produção e logística, considerando as restrições tecnológicas envolvidas e obtendo soluções otimizadas em termos de custos e margens de contribuição ao lucro. Quando estas abordagens são implementadas em sistemas de apoio a decisões, proporcionam ao tomador de decisão poder de análise e flexibilidade para replanejar quando houver mudanças nos parâmetros dos problemas, bem como gerar e avaliar cenários alternativos. Diversos exemplos de aplicações práticas de sucesso das abordagens de pesquisa operacional em problemas de planejamento agregado da produção e logística podem ser encontrados, por exemplo, no site www.scienceofbetter.org do Institute for Operations Research and the Management Sciences (INFORMS).

No Brasil, alguns exemplos de trabalhos aplicando técnicas de pesquisa operacional em problemas relacionados ao planejamento de cadeias agroindustriais e projeto de redes logísticas podem ser encontrados em Taube (1996), no planejamento integrado da produção de carne avícola, em Yoshizaki et al. (1996), na distribuição descentralizada de etanol, em Munhoz e Morabito (2001), na mistura e distribuição de suco concentrado congelado de laranja, em Caixeta-Filho et al. (2002), na produção e comercialização de flores, em Colin et al. (1999), Kawamura et al. (2005) e Paiva e Morabito (2007), no planejamento da produção, estocagem e distribuição de açúcar e álcool, entre outros.

Poucos trabalhos foram encontrados na literatura estudando o planejamento tático-estratégico da produção e logística no setor de sementes de milho (veja, por exemplo, JONES et al., 2003). Recentemente, Junqueira e Morabito (2006) propuseram um modelo de otimização linear que busca minimizar o custo total de produção e logística, incluindo os custos de transporte entre regiões agrícolas, unidades industriais e de entrega, os custos de processamento e os custos fiscais de imposto de circulação de mercadorias e serviços (ICMS), ao longo do período de planejamento. Redes produtoras de sementes de milho, em geral, possuem vários campos de produção agrícola, diversas unidades industriais (Unidades de Beneficiamento de Sementes - UBS) e muitos pontos de demanda. Associadas às UBS, são relevantes as restrições de capacidade dos processos de produção envolvidos 
(e.g., preparação e beneficiamento das sementes). Além disso, a programação de colheita e a previsão de demanda dos produtos devem ser atendidas. Junqueira e Morabito (2006) testaram este modelo apenas em situações simplificadas da realidade.

No presente trabalho analisamos a aplicação deste modelo de planejamento otimizado em um estudo de caso de uma safra completa de uma empresa do setor de sementes de milho. Neste setor, empresas que experimentam um crescimento rápido de sua rede produtora de sementes de milho tendem a criar regras práticas para realizar o planejamento agregado da produção e logística. Por exemplo, algumas empresas focam apenas algumas variáveis, como as distâncias do campo às UBS, mas deixam outras variáveis subotimizadas, como o transporte das UBS às regiões de demanda e os custos fiscais inerentes à movimentação e capacidade das unidades.

Este artigo está organizado da seguinte maneira: a seção 2 faz uma breve análise do setor de sementes de milho e a seção 3 discute o caso de uma empresa estudada deste setor, analisando-a sob diversos aspectos. Na seção 4 estuda-se a aplicação do modelo de planejamento otimizado em uma safra completa desta empresa. Para isso, foram gerados e avaliados diferentes cenários por meio deste modelo, considerando os custos de ICMS e de secagem, e comparados ao método utilizado pela empresa. Os resultados mostram que reduções importantes de custos podem ser obtidas nos planos de produção e logística, quando consideradas as premissas presentes no modelo. Finalmente, a seção 5 apresenta as considerações finais deste estudo e perspectivas para pesquisa futura.

\section{Setor de sementes de milho}

Slack et al. (2002) definem estratégia competitiva como sendo o padrão global de decisões e ações que posicionam a organização em seu ambiente, com o objetivo de fazê-la atingir seus objetivos no longo prazo. Já competências organizacionais podem ser definidas como recursos intangíveis que, em relação aos concorrentes, são difíceis de ser imitadas. Em relação a mercados e clientes, são essenciais para prover produtos e serviços diferenciados, e em relação ao processo de mudança e evolução da empresa, é o fator fundamental que dá flexibilidade e que permite a exploração de novos mercados (PRAHALAD; HAMEL apud FLEURY; FLEURY, 2003). Fleury e Fleury (2003) consideram que qualquer estratégia competitiva genericamente pode se encaixar dentre uma das seguintes categorias: i) excelência operacional; ii) inovação em produto; e iii) relação com o cliente. Já as competências organizacionais podem ser agrupadas em três funções principais: i) operações; ii) pesquisa, desenvolvimento e engenharia; e iii) marketing e vendas. As características destas estratégias podem ser visualizadas na Tabela 1 .

Santini (2002) destaca as estratégias mais utilizadas no setor de sementes. As principais são: i) fusão e aquisição de empresas nacionais avançadas em pesquisa e desenvolvimento, como forma de quebrar barreiras de entrada; ii) contratos de parceria tecnológica com a finalidade de desenvolvimento de linhagens mais ajustadas aos diferentes climas e ambientes de produção; iii) diversificação das empresas agroquímicas em direção ao segmento de sementes, visando oferecer pacotes tecnológicos "semente + defensivo", aumentando a produtividade desses produtos; iv) investimento em assistência técnica, distribuição e comercialização, que são ativos específicos, complementando o pacote tecnológico com uma componente de serviços; v) políticas defensivas que aguardam as empresas líderes em pesquisa e desenvolvimento de produtos lançarem seus produtos no mercado, para então investir em desenvolvimento, reduzindo o risco por já conhecer algumas respostas do mercado e detalhes do processo; vi) investimento em marca e publicidade visando maximizar o preço de seus produtos, diferenciando-os; vii) integração vertical para aumentar o controle da qualidade, reduzir custos e manter o segredo biotecnológico; e viii) localização das UBS visando em alguns casos a minimização de custos de transporte e em outros a proximidade do cliente.

No estudo de caso realizado em sua pesquisa, Santini (2002) avaliou a importância dada pelas empresas estudadas a cada vantagem competitiva, atribuindo o valor 1 quando a vantagem não tem nenhuma importância, 2, quando tem pouca importância e 3 , quando tem muita importância (Tabela 2). Pode-se verificar pelas médias dos valores atribuídos a cada vantagem competitiva, uma

Tabela 1. Características das estratégias competitivas. (FLEURY; FLEURY, 2003*).

\begin{tabular}{llll}
\hline \multicolumn{1}{c}{ Estratégia } & \multicolumn{1}{c}{ Excelência operacional } & \multicolumn{1}{c}{ Inovação em produto } & \multicolumn{1}{c}{ Orientação para o cliente } \\
\hline Função crítica & Operações & $\begin{array}{l}\text { Pesquisa, desenvolvimento e enge- } \\
\text { nharia (P\&D\&E). }\end{array}$ & Marketing e vendas (M\&V). \\
Mercado alvo & Mercados que valorizam quali- & $\begin{array}{l}\text { Mercados aptos a absorver produtos } \\
\text { dade e preço. }\end{array}$ & $\begin{array}{l}\text { Atendimento de necessidades presen- } \\
\text { Fonte de lucratividade e futuras de clientes específicos. }\end{array}$ \\
& $\begin{array}{l}\text { Altas margens por produto e } \\
\text { economias de escala. }\end{array}$ & $\begin{array}{l}\text { Alta lucratividade que usufruem } \\
\text { durante o período que possuem } \\
\text { posição de monopólio. }\end{array}$ & $\begin{array}{l}\text { Maximização do valor do produto } \\
\text { final atendendo necessidades espe- } \\
\text { cíficas. }\end{array}$ \\
\hline
\end{tabular}

*Adaptado 
grande importância dada a contratos de parceria tecnológica e tecnologia/inovação, que são vantagens obtidas por meio da função de P\&D\&E (Tabela 1). Em segundo grau de importância vêm a rede de comercialização e a rede de assistência técnica, relacionadas à função de $\mathrm{M} \& \mathrm{~V}$. Por fim, aparecem as vantagens de economia de escala, fornecedores de insumos, economia de escopo e localização das UBS, relacionadas à função de operações.

Na Tabela 3, as vantagens competitivas da Tabela 2 são agrupadas com as funções que teriam uma relação mais direta para sua obtenção. Desta análise, verificamos que as empresas estudadas na Tabela 2 atribuem grande importância à função de P\&D\&E. Entretanto, para as funções de $M \& V$ e operações, com exceção da empresa 4, as outras empresas atribuem maior importância à função de $M \& V$.

Com base na discussão das Tabelas 1 a 3, percebe-se que as empresas do setor de sementes de milho tendem a adotar a estratégia de inovação em produto, cuja função de P\&D\&E adquire o papel de competência central, coordenando as demais funções estratégicas. A função de $\mathrm{M} \& \mathrm{~V}$, representada pela rede de comercialização e assistência técnica, dessa forma, assume o papel de negociador no lançamento de novos produtos, embora também esteja próxima do produtor rural, entendendo seu comportamento, suas necessidades e tendências. Fleury e Fleury (2003), ao caracterizarem a estratégia em questão, não especificam as atribuições da função de operações. Entre-

Tabela 2. Importância das vantagens competitivas no ambiente concorrencial do mercado de sementes de milho. (SANTINI, 2002*).

\begin{tabular}{|c|c|c|c|c|c|c|}
\hline \multirow[t]{2}{*}{ Vantagem competitiva } & \multicolumn{5}{|c|}{ Empresa } & \multirow[t]{2}{*}{ Média } \\
\hline & 1 & 2 & 3 & 4 & 5 & \\
\hline $\begin{array}{l}\text { Contratos de parceria } \\
\text { tecnológica }\end{array}$ & 3,0 & 3,0 & 3,0 & 3,0 & 3,0 & 3,0 \\
\hline Tecnologia/inovação & 3,0 & 3,0 & 3,0 & 3,0 & 3,0 & 3,0 \\
\hline Rede de comercialização & 3,0 & 1,0 & 3,0 & 2,0 & 3,0 & 2,8 \\
\hline Rede de assistência técnica & 3,0 & 3,0 & 3,0 & 2,0 & 2,0 & 2,8 \\
\hline Economia de escala & 3,0 & 2,0 & 3,0 & 3,0 & 2,0 & 2,6 \\
\hline Fornecedores de insumos & 2,0 & 2,0 & 3,0 & 2,0 & 2,0 & 2,2 \\
\hline Economia de escopo & 1,0 & 2,0 & 3,0 & 2,0 & 2,0 & 2,0 \\
\hline Localização da UBS & 3,0 & 1,0 & 1,0 & 3,0 & 2,0 & 2,0 \\
\hline
\end{tabular}

Tabela 3. Importância das funções estratégicas no ambiente concorrencial do mercado de sementes de milho.

\begin{tabular}{lccccc}
\hline \multicolumn{1}{c}{ Função estratégica } & \multicolumn{5}{c}{ Empresa } \\
\cline { 2 - 6 } & $\mathbf{1}$ & $\mathbf{2}$ & $\mathbf{3}$ & $\mathbf{4}$ & $\mathbf{5}$ \\
\hline $\begin{array}{l}\text { Pesquisa, desenvolvimento } \\
\text { e engenharia }\end{array}$ & 3,00 & 3,00 & 3,00 & 3,00 & 3,00 \\
Marketing e vendas & 3,00 & 2,00 & 3,00 & 2,00 & 2,50 \\
Operações & 2,25 & 1,75 & 2,50 & 2,50 & 2,00 \\
\hline
\end{tabular}

tanto, analisando a cadeia de produção, pode-se verificar um papel relevante desta função.

Primeiramente, as economias de escala propiciam a diluição do investimento em P\&D\&E. Entretanto, o aumento no volume de produção deixa a operação mais complexa, especialmente se a carteira de produtos é grande. Para não perder eficiência obtendo estas vantagens, tornam-se primordiais o planejamento otimizado da produção e da logística, em conjunto com uma gestão eficiente do portfólio de produtos.

Além disso, a inovação em produtos visa a lucratividade nos períodos em que o produto inovador possui monopólio de mercado, ou seja, alto preço de venda. Dessa forma, como os custos fiscais são dependentes desse preço, a utilização de rotas com tarifas de ICMS favoráveis na distribuição pode trazer grandes ganhos econômicos. Para isso, o planejamento da produção e da logística, incluindo custos de ICMS, como sugerem Yoshizaki (2002), Junqueira e Morabito (2006), poderia trazer importantes contribuições.

Ainda, com a ênfase em inovação, passa a ser mais freqüente o lançamento de novos produtos. Com isso, a função de previsão de demanda passa a ser mais complexa, pois não se conhece o comportamento do mercado em relação ao novo produto. Isso gera maior grau de incerteza na demanda. Sendo assim, dadas as incertezas tanto no suprimento quanto na demanda, o planejamento da produção e da logística deve ser refeito no médio e curto prazo, além de estar estruturado de forma a considerar as questões mencionadas acima, para ser eficaz e adaptar-se às mudanças no ambiente.

\section{Estudo de caso}

Neste trabalho foi estudado o caso de uma empresa do setor de sementes de milho de capital nacional, com áreas de produção agrícola próprias nos estados de São Paulo, Minas Gerais e Goiás. Esta empresa, aqui denominada empresa A, possui UBS em São Paulo, Minas Gerais e Goiás e atende ao mercado no âmbito nacional. O contato com a empresa se deu durante o desenvolvimento de um projeto de melhoramento do seu sistema de produção e logística durante o período de 2001 a 2003, quando um dos autores fazia parte da equipe de um projeto de consultoria contratada. Como fontes de informações, foram utilizados documentos e dados recolhidos durante este período do projeto.

A produção agrícola era realizada em dois sistemas: com terra própria e com cooperantes. Para o caso da terra própria, a empresa tem total controle sobre a operação e tecnologia utilizada. Este sistema é utilizado em mais de 90\% das áreas. Para o caso de cooperantes, a empresa define os produtos a serem plantados, fornece a tecnologia de produção (as sementes básicas e os procedimentos 
agrícolas e industriais para a obtenção do produto final), e acompanha a lavoura, realizando os tratos culturais e a colheita. O milho colhido é entregue na UBS, onde é transformado em sementes e encaminhado para a comercialização.

A produção dos cooperantes é realizada 100\% irrigada, por meio de pivôs. Das terras próprias, aproximadamente $80 \%$ é irrigada. O despendoamento ocorre $100 \%$ manual. Esta operação consiste na retirada do pendão da espécie fêmea, na qual se desenvolverá a semente a partir do cruzamento com o grão de pólen da espécie macho (hibridação). Quando se cruzam duas variedades, obtêm-se híbridos simples, cujas sementes geram plantas com alta produtividade com a aplicação intensiva de insumos. Os híbridos duplos são formados pelo cruzamento de uma variedade e um híbrido simples. Eles geram plantas com baixa produtividade, embora maior que a da variedade pura, e não necessitam de grandes quantidades de insumos. Os híbridos triplos têm um desempenho intermediário em relação aos híbridos simples e duplos, consistindo no cruzamento de um híbrido duplo e uma variedade ou dois híbridos simples. A colheita de $100 \%$ das áreas irrigadas, além de grande parte da colheita de sequeiro (não irrigada), é realizada em espigas; entretanto, o restante ocorre com colheita mecânica em grãos. Em geral, são colhidos em grãos as variedades e os híbridos duplos com menor valor agregado. Para mais detalhes do processo produtivo de sementes de milho, veja, por exemplo, Carvalho e Nakagawa (2000) e Junqueira (2006).

O transporte do campo para as UBS é inteiramente contratado e, em geral, é realizado pelo mesmo empreiteiro que faz a colheita. Como uma parte da matéria-prima chega na UBS do campo em grãos, as UBS possuem toda a infra-estrutura para secar grãos úmidos. A armazenagem de grãos secos e não beneficiados é feita em big bags, silos internos ou externos. A transferência desse material acontece para ajustes entre produção e demanda, bem como para a redução de custos de produção e transporte. A armazenagem e transferência em big bags é um procedimento praticado, e muitas vezes as peneiras com menor valor comercial são encaminhadas para outros destinos.

A carteira de produtos é liderada por híbridos duplos e também possui uma parcela com variedades, que atinge aos produtores com grau de tecnificação de médio a baixo, veja, por exemplo, Giordano (1993), para entender a segmentação do mercado de sementes de milho. Os híbridos simples e triplos também possuem uma parcela na carteira e há perspectivas de desenvolvimento de novas espécies desses tipos. As práticas de vendas são agressivas, adotando-se vendas em consignação, entregando, na medida do possível, as peneiras demandadas pelas regiões mercadológicas.

Em suma, a empresa A possui estratégias operacionais e de vendas agressivas. Em termos de operações, integra totalmente a cadeia de produção, sendo muito comum no setor ter o cooperante. Ela possui suas áreas de produção agrícola próximas das UBS, ao passo que quando o cooperante está envolvido na cadeia, isto se torna mais difícil. Ela utiliza frequientemente transferência de produtos semiprocessados entre as UBS, uma vez que a prática mais usual é ensacar o produto final com um mínimo de armazenamento intermediário. Já em termos de vendas, utiliza a venda sob consignação e diferenciação através de peneiras, táticas pouco utilizadas no setor.

Dessa forma, a empresa A pode ser caracterizada como a que utiliza a estratégia de excelência operacional, tendo como competência central a função de produção, apresentando uma posição estratégica diferente de outras empresas do setor (seção 2). Convém observar que, durante o presente estudo, outras empresas foram visitadas e, diferentemente da empresa A, utilizam a estratégia de inovação do produto, tendo como competência central a função de desenvolvimento de produto, bastante alinhada à tendência do setor. A Tabela 4 mostra a caracterização das funções estratégicas da empresa A.

Sendo assim, para a empresa A, um planejamento da produção e logística otimizado contribui diretamente para o sucesso de sua estratégia competitiva. Por meio desse planejamento, do conhecimento da realidade operacional da empresa que ele proporciona através de simulações e análise de custos marginais, bem como do seu acompanhamento e revisões do plano, a empresa tem a possibilidade de otimizar seus recursos produtivos, minimizando custos e maximizando receitas. Convém notar que para outras empresas do setor (como as mencionadas acima), o planejamento traz as vantagens ressaltadas na seção 2, auxilia a manter a eficiência operacional com grandes volumes de produção e carteira de produtos diversificada, proporciona redução de custo através do planejamento tributário com o ICMS para os produtos com alto valor agregado e possibilita uma reação rápida com modificações na previsão de demanda. Para as empresas, mais do que a determinação de soluções, a elaboração deste planejamento e a análise de seus custos marginais objetivam a capacitação da equipe gerencial a entender padrões, comportamentos e tendências inerentes à cadeia de produção.

Tabela 4. Comparação entre as funções estratégicas da empresa A.

\section{Empresa A}

Estratégia competitiva Excelência operacional.

Desenvolvimento de Em fase de estruturação, a empresa foca produto desenvolvimentos incrementais.

Produção

Baixos custos operacionais é a fonte de lucratividade e possui flexibilidade.

Vendas Apóia-se em estratégias agressivas para aumentar o market share. 


\section{Aplicação do modelo}

Nesta seção estuda-se a aplicação do modelo de planejamento otimizado de produção e logística de sementes de milho à realidade da empresa A. Este modelo, apresentado resumidamente no Apêndice por conveniência, representa um problema de planejamento agregado para decisões táticas em dois estágios (níveis), sendo o primeiro deles relativo às operações de colheita, transporte e preparo das sementes de milho e o outro, às operações de transporte e beneficiamento. Há possibilidade de transbordo entre os estágios, representada pelas transferências de grãos após o preparo das sementes. Os recursos dos estágios são limitados, tratando-se, portanto, de restrições de capacidade de processo de produção e logística. Como é considerada mais de uma UBS em diferentes locais, pode ser caracterizado como um problema multiplantas. Além disso, é considerado mais de um produto (problema multiproduto), e mais de um período no horizonte de decisão (problema multiperíodo).

O modelo de otimização determina as quantidades de cada produto a serem transportadas das unidades de produção agrícola para as UBS, partindo de uma programação de colheita. Estabelece também as quantidades a serem processadas em cada diferente etapa da UBS (secagem e beneficiamento), respeitando limitações de capacidade destes processos de produção, bem como níveis de estoque estabelecidos. E determina se há transferências entre unidades e se atende à demanda distribuída em diferentes regiões. Para garantir a eficiência econômica do plano, é necessário considerar os custos de transporte, processamento e fiscais (ICMS), dependendo do caminho que o produto percorre pela rede. Para mais detalhes do modelo de otimização, além dos apresentados no Apêndice, os leitores podem consultar Junqueira e Morabito (2006).

\subsection{Levantamento de dados}

O levantamento de dados foi feito ao longo de 2002. Este levantamento traz dados referentes às sementes produzidas para atender à demanda da safra 2003/2004. Os períodos considerados são os utilizados pela empresa A para realizar a previsão da demanda, e estão resumidos na Tabela 5. A data de término indica o prazo máximo

Tabela 5. Períodos de comercialização.

\begin{tabular}{cccc}
\hline Período & Início & Término & Duração (dias) \\
\hline Per 1 & $01 / 04 / 03$ & $30 / 06 / 03$ & 91 \\
Per 2 & $01 / 07 / 03$ & $31 / 07 / 03$ & 31 \\
Per 3 & $01 / 08 / 03$ & $31 / 08 / 03$ & 31 \\
Per 4 & $01 / 09 / 03$ & $15 / 10 / 03$ & 45 \\
Per 5 & $16 / 10 / 03$ & $15 / 11 / 03$ & 31 \\
Per 6 & $16 / 11 / 03$ & $15 / 01 / 04$ & 61 \\
Per 7 & $16 / 01 / 04$ & $31 / 01 / 04$ & 16 \\
\hline
\end{tabular}

para o departamento de produção entregar as quantidades do produto presentes na previsão da demanda. A carteira de produtos utilizada pela empresa A na safra 2003/2004, juntamente com os respectivos preços de venda são dados pela Tabela 6. Para preservar interesses da empresa, todos os valores monetários de preços e custos foram distorcidos mantendo-se certa proporcionalidade; portanto, estas unidades são aqui referidas simplesmente como unidades monetárias. Na Tabela 6, HD indica híbrido duplo, HS, híbrido simples, HT, híbrido triplo e VAR, variedade. A configuração da rede logística da empresa A utilizada no modelo é apresentada na Figura 1.

Conforme pode ser observado na Figura 1, a região agrícola de Goiás foi dividida em 4 , já que os custos de cada uma em relação à UBS são bastante diferenciados. Mais especificamente, a região de São Paulo está nos arredores de Barretos, GO1, na região de Itumbiara, GO2, na de Rio Verde, GO3 e na de Cristalina GO4. Com exceção da UBS GO1, que possui apenas preparo de grãos, todas as outras UBS possuem preparo de grãos e espigas. Todas as UBS possuem beneficiamento. As regiões de demanda estão agrupadas de acordo com as maiores regiões de demanda. A divisão entre elas obedece ao requisito

Tabela 6. Carteira de produtos.

\begin{tabular}{cc}
\hline Período & Preço de venda (\$) \\
\hline HD1 & 45,00 \\
HD2 & 48,00 \\
HD3 & 48,00 \\
HS1 & 70,00 \\
HS2 & 90,00 \\
HS3 & 90,00 \\
HT1 & 60,00 \\
HT2 & 62,00 \\
HT3 & 60,00 \\
VAR & 19,00 \\
\hline
\end{tabular}

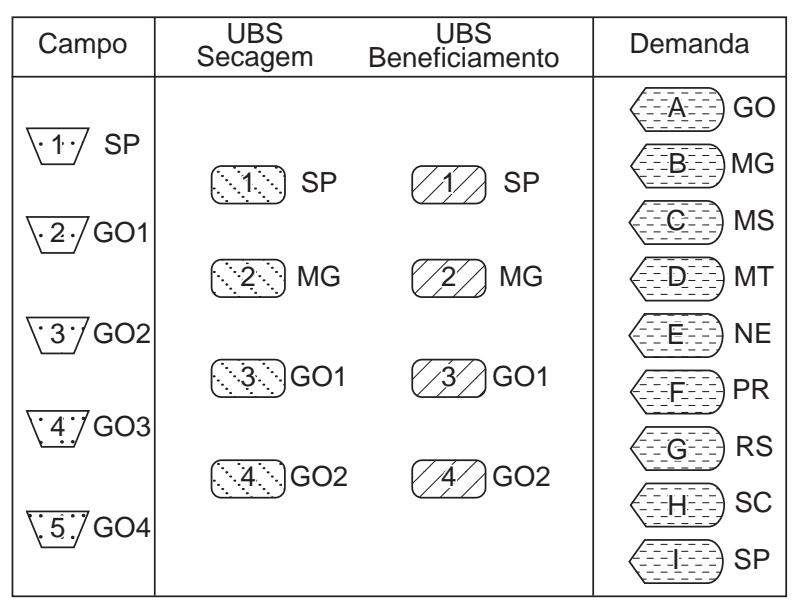

Figura 1. Configuração da rede logística da Empresa A. 
mínimo (JUNQUEIRA; MORABITO, 2006) que é o de separar as áreas com regras fiscais diferentes. No entanto, elas agrupam extensões territoriais muito grandes como o estado do Mato Grosso e a região Nordeste, podendo causar distorções nos custos de transporte ao usar o dado médio.

A Figura 2 mostra a previsão da demanda da empresa A para a safra 2003/2004 por produto e período de comercialização. A figura evidencia os picos de demanda nos períodos 1 e 2 devido à safra de verão, e no período 6 devido à safrinha. Algumas regiões, devido a suas características climáticas, possibilitam o cultivo de duas culturas ao ano, sendo o segundo cultivo chamado de safrinha. Já a Figura 3 mostra a demanda por produto. Nela, percebe-se que o HD1 é o principal produto, seguido pelo HT1. Cabe ressaltar que, as escalas dos gráficos de previsão da demanda e programação de colheita, foram retiradas por motivos de confidenciabilidade, para preservar as informações fornecidas pela empresa $\mathrm{A}$.

A Figura 4 mostra a previsão de demanda por produto e região. Nela, pode-se observar que a região com maior
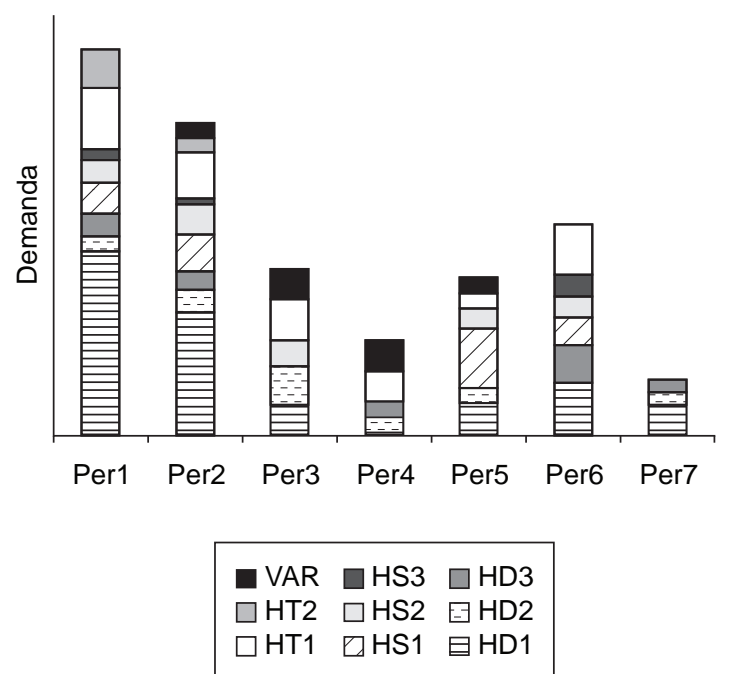

Figura 2. Previsão da demanda da empresa A por período.

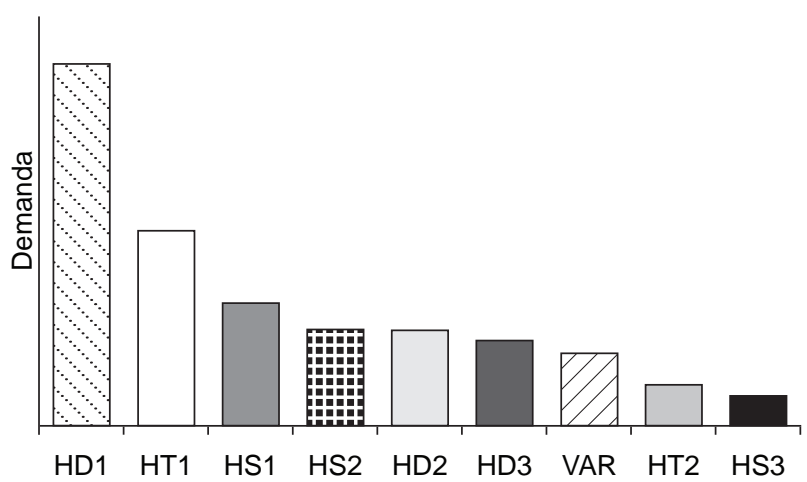

Figura 3. Previsão da demanda da empresa A por produto. demanda é o RS. No entanto, há predomínio de híbridos duplos, indicando que o cliente é basicamente agricultor dos segmentos C e D. Em seguida vem o Estado do Paraná, onde há uma distribuição maior de híbridos triplos, indicando que também há clientes do segmento B. Já no Estado do Mato Grosso, percebe-se uma grande quantidade de híbridos simples, indicando o predomínio de agricultores tecnificados.

A Figura 5 mostra a programação de colheita por produto e período da empresa A para a safra 2003/2004. Analisando este gráfico, pode-se perceber que a maior parte da produção está concentrada no período 1, pois inclui a produção de sequeiro, principalmente para os híbridos duplos e variedades. Já a Figura 6 mostra a programação de colheita por produto e região agrícola. A figura mostra que nas regiões GO2 e GO3 está concentrada a maior parte da produção. A região SP responde por uma parcela pequena da produção total.

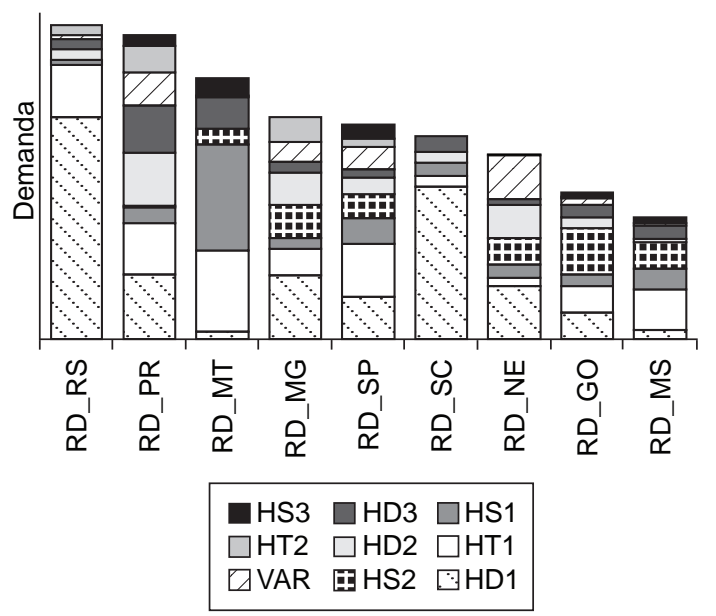

Figura 4. Previsão da demanda da empresa A por região.

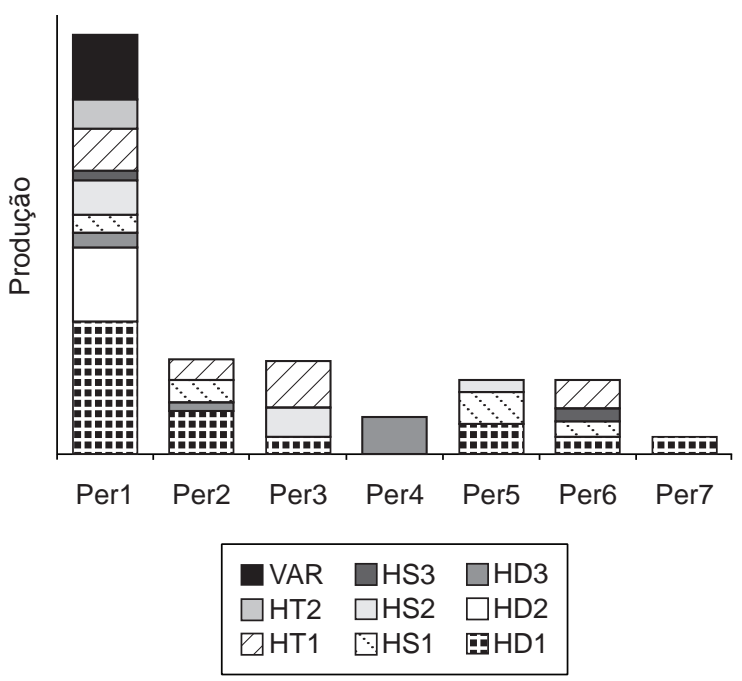

Figura 5. Programação de colheita da empresa A por período. 
Cabe ressaltar que quando os dados de programação de colheita e previsão de demanda foram confrontados, houve períodos em que a produção não era suficiente para atender a demanda, tornando o planejamento infactível. Esta diferença ocorre devido às incertezas da produtividade e época de colheita da produção agrícola, e incertezas quanto à quantidade e época de entrega da previsão de demanda. Para ajustar isso, a quantidade faltante foi abatida da previsão da demanda.

A Tabela 7 mostra a capacidade de produção diária das UBS, em sacos de $20 \mathrm{~kg}$ por dia, por tipo de recurso: preparo de grãos e espigas, armazenagem de grãos secos, beneficiamento e armazenagem de produto acabado. Analisando esta tabela, percebe-se que a UBS-MG é a que possui maior capacidade de preparo de espigas e a de SP possui a menor capacidade. Já a UBS-GO1 é a que tem maior capacidade de processamento de grãos, seguida pela UBS-MG. Em termos de armazenagem de grãos secos, a UBS-SP é a que tem maior capacidade, enquanto que a menor é em GO1. A capacidade de beneficiamento é a mesma para todas as unidades. Já a maior armazenagem de produto acabado ocorre na UBS-GO1.

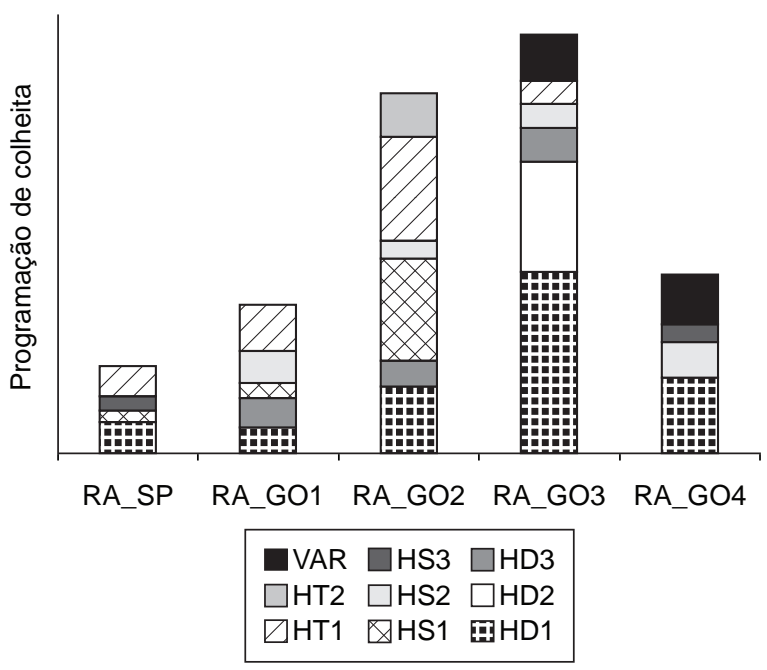

Figura 6. Programação de colheita da empresa A por região agrícola.

Tabela 7. Capacidade das UBS (saco de $20 \mathrm{~kg}$ por dia).

\begin{tabular}{lcccc}
\hline & \multicolumn{4}{c}{ UBS } \\
\cline { 2 - 5 } \multicolumn{1}{c}{ Tipo } & GO1 & GO2 & MG & SP \\
\hline Preparo (grãos) & 16.000 & 9.750 & 12.000 & 9.750 \\
Preparo (espigas) & 0 & 1.636 & 2.231 & 885 \\
Armazenagem do grão & 1.650 & 4.800 & 2.888 & 6.000 \\
seco & & & & \\
Beneficiamento & 3.214 & 3.214 & 3.214 & 3.214 \\
Armazenagem do & 7.104 & 3.042 & 3.870 & 3.120 \\
produto acabado & & & & \\
\hline
\end{tabular}

Para todas as UBS, a capacidade de preparo é superior à de beneficiamento; no entanto, cabe lembrar que não necessariamente o beneficiamento é o gargalo do sistema produtivo, já que o preparo depende do tempo de ciclo de secagem, caracterizando um fluxo em batelada, enquanto que o beneficiamento possui fluxo contínuo.

Observa-se que o preparo de grãos, armazenagem de grãos secos e armazenagem de produto acabado são recursos que, além de processarem e armazenarem sementes de milho, são utilizados por outras culturas como soja na UBS-GO1 e sorgo na UBS-MG. Além disso, o preparo de espigas concorre com o de sementes básicas na UBS-SP; todavia as quantidades são desprezíveis se comparadas ao preparo de espigas para sementes comerciais.

A Tabela 8 mostra o custo de transporte de matériaprima entre a região agrícola e a UBS de preparo de matéria-prima, separado por tipo de colheita. A Tabela 9 mostra os custos de transporte de grãos secos entre UBS de preparo de matéria-prima e UBS de beneficiamento. A Tabela 10 mostra os custos de transporte de produto acabado entre UBS de beneficiamento e UBS região de demanda. Todos estes valores foram fornecidos pelo responsável da empresa $\mathrm{A}$, especialista na contratação desse transporte.

Foi considerada uma redução de custo de $\$ 1,5$ unidades monetárias por saco de $20 \mathrm{~kg}$ para o preparo de espigas na UBS de Minas Gerais, conforme informado pela administração da empresa A. Isso ocorre graças ao secador aquecido por sabugo de milho ao invés de gás, que é o combustível utilizado nas outras UBS. O rendimento na secagem foi de $50 \%$ para espigas e $96 \%$ para grãos. Já o rendimento no beneficiamento foi de $85 \%$, conforme informado pelo departamento técnico da empresa $\mathrm{A}$.

\subsection{Resultados}

Os dados apresentados na seção 4.1 foram usados no modelo de otimização linear do Apêndice. Para resolver o modelo, foi utilizada a linguagem de modelagem GAMS 2.0.10.0 com o software de otimização CPLEX 7.0.0, em um microcomputador Toshiba Satellite A10, com processador Intel-Pentium 4 de 2,20 GHz, com memória RAM utilizável de 496 MB e HD de 40 GB. O modelo original resultou em 4.758 equações e 6.679 variáveis. A Tabela 11 mostra a manipulação da matriz dos coeficientes tecnológicos do modelo realizada por meio do algoritmo de preparação do CPLEX. Ao analisá-la, pode-se concluir que a matriz possui várias equações com dependência linear; note que das 4.758 equações originais, apenas 625 delas realmente restringem a solução do problema. Os tempos computacionais (em segundos) requeridos para resolver cada cenário (definidos adiante) pelo GAMS/ CPLEX estão apresentados na Tabela 12. Note que estes tempos são relativamente pequenos e bem aceitáveis para 
Tabela 8. Custos de transporte de matéria-prima (\$/t).

\begin{tabular}{|c|c|c|c|c|c|c|}
\hline \multirow{2}{*}{\multicolumn{2}{|c|}{$\begin{array}{c}\text { UBS } \\
\text { Secagem }\end{array}$}} & \multicolumn{5}{|c|}{ Campo de Produção } \\
\hline & & RA_GO1 (\$) & RA_GO2 (\$) & RA_GO3 (\$) & RA_GO4 (\$) & RA_SP (\$) \\
\hline \multirow{4}{*}{ 跷 } & UBS_GO1 & 32,50 & 33,50 & 3,00 & 7,00 & 30,00 \\
\hline & UBS_GO2 & 13,00 & 10,00 & 33,50 & 33,50 & 31,00 \\
\hline & UBS_MG & 13,00 & 13,00 & 32,50 & 32,50 & 25,00 \\
\hline & UBS_SP & 32,50 & 35,00 & 33,50 & 33,50 & 10,00 \\
\hline \multirow{4}{*}{ 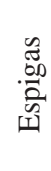 } & UBS_GO1 & 32,50 & 35,00 & 3,00 & 7,00 & 35,00 \\
\hline & UBS_GO2 & 15,00 & 10,00 & 37,50 & 37,50 & 35,00 \\
\hline & UBS_MG & 15,00 & 15,00 & 34,50 & 34,50 & 29,00 \\
\hline & UBS_SP & 32,50 & 40,00 & 37,50 & 37,50 & 10,00 \\
\hline
\end{tabular}

Tabela 9. Custos de transporte de transferência (\$/t).

\begin{tabular}{lcccc}
\hline UBS destino & \multicolumn{4}{c}{ UBS origem } \\
\cline { 2 - 5 } & UBS_GO1 & UBS_GO2 & UBS_MG & UBS_SP \\
& $\mathbf{( \$ )}$ & $\mathbf{( \$ )}$ & $\mathbf{( \$ )}$ & $\mathbf{( \$ )}$ \\
\hline UBS_GO1 & - & 35,00 & 35,00 & 35,00 \\
UBS_GO2 & 35,00 & - & 30,00 & 35,00 \\
UBS_MG & 35,00 & 30,00 & - & 30,00 \\
UBS_SP & 35,00 & 35,00 & 30,00 & - \\
\hline
\end{tabular}

Tabela 10. Custos de transporte de entrega (\$/saco $20 \mathrm{~kg})$.

\begin{tabular}{lcccc}
\hline Regiões & \multicolumn{4}{c}{ UBS Origem } \\
\cline { 2 - 5 } & $\begin{array}{c}\text { UBS_GO1 } \\
\mathbf{( \$ )}\end{array}$ & $\begin{array}{c}\text { UBS_GO2 } \\
\mathbf{( \$ )}\end{array}$ & $\begin{array}{c}\text { UBS_MG } \\
\mathbf{( \$ )}\end{array}$ & $\begin{array}{c}\text { UBS_SP } \\
\mathbf{( \$ )}\end{array}$ \\
\hline RD_GO & 0,65 & 0,65 & 1,18 & 1,40 \\
RD_MG & 1,15 & 1,15 & 0,72 & 0,93 \\
RD_MS & 1,10 & 1,10 & 1,10 & 1,10 \\
RD_MT & 1,60 & 1,30 & 1,30 & 1,30 \\
RD_NE & 3,00 & 3,00 & 3,00 & 3,00 \\
RD_PR & 1,10 & 1,10 & 1,10 & 0,68 \\
RD_RS & 1,35 & 1,35 & 1,35 & 1,15 \\
RD_SC & 1,35 & 1,35 & 1,35 & 1,05 \\
RD_SP & 1,40 & 1,40 & 0,93 & 0,56 \\
\hline
\end{tabular}

Tabela 11. Matriz dos coeficientes tecnológicos.

\begin{tabular}{lcc}
\hline & Linhas & Colunas \\
\hline Original & 4.758 & 6.679 \\
Eliminado & 4.001 & 4.354 \\
LP Reduzido & 625 & 2.138 \\
\hline
\end{tabular}

Tabela 12. Tempos computacionais (em segundos).

\begin{tabular}{lcccc}
\hline & s/ICMS & c/ICMS & c/prep & c/ICMS + prep \\
\hline Presolve & 0,04 & 0,03 & 0,03 & 0,03 \\
Geração & 0,5 & 0,22 & 0,241 & 0,22 \\
Execução & 0,5 & 0,22 & 0,241 & 0,22 \\
Iterações & 380 & 381 & 360 & 410 \\
\hline
\end{tabular}

apoiar as decisões na prática, proporcionando uma grande agilidade para gerar e avaliar diferentes cenários.

Conforme mencionado, foram gerados diferentes cenários considerando os custos de ICMS e secagem diferenciados, comparando-os com os custos obtidos pela regra empregada pela empresa A. Esta regra consiste no envio de sementes para a UBS mais próxima da região agrícola, representada no modelo da seguinte maneira: i) considera-se no modelo apenas os custos de transportes de matéria-prima, no qual os menores custos representam as menores distâncias; ii) os custos de transferência foram substituídos por valores suficientemente grandes, tal que o modelo seja forçado a não optar por transferir grãos secos; e iii) os custos de transporte na entrega foram substituídos por valores suficientemente grandes, tal que o produto acabado possa ser entregue a partir da UBS mais próxima; todavia, sem interferir na decisão de envio de sementes do campo para a UBS.

Para melhor compreensão das componentes de custo propostas para a função objetivo, são gerados cenários distintos, ora isolando o efeito das componentes de ICMS e do custo adicional de preparo de matéria-prima, ora integrando-as. Foram realizadas três comparações entre os custos obtidos pelo método utilizado pela empresa A e o modelo de otimização utilizado neste trabalho. $\mathrm{Na}$ primeira comparação, o modelo contempla os custos de transporte e os custos de ICMS. Na segunda, contempla os custos de transporte e o custo adicional de preparo de espigas nas UBS de GO e SP. A terceira comparação agrega os três custos na função objetivo: transporte, adicional de preparo de espigas e ICMS. Por meio destas análises, pretende-se verificar o efeito isolado das componentes da função objetivo, bem como a combinação delas.

A Tabela 13 representa a comparação entre o método empregado pela empresa A e o modelo proposto, considerando apenas os custos de ICMS. As colunas "Método da empresa" e "c/ ICMS" representam o percentual de cada uma das parcelas de custo no custo total. Já a coluna da diferença representa o percentual de perda ou ganho com o método proposto em relação ao método da 
Tabela 13. Comparação entre cenário anterior e o proposto considerando o ICMS

\begin{tabular}{lccc}
\hline & $\begin{array}{c}\text { Método empresa } \\
(\boldsymbol{\%})\end{array}$ & $\begin{array}{c}\text { c/ICMS } \\
(\boldsymbol{\%})\end{array}$ & $\begin{array}{c}\text { Diferença } \\
(\boldsymbol{\%})\end{array}$ \\
\hline Transporte MP & 15 & 20 & 20 \\
Preparo MP & 0 & 0 & \\
Transferência & 0 & 1 & \\
Entrega & 33 & 36 & -3 \\
ICMS & 53 & 43 & -27 \\
Total & 100 & 100 & -12 \\
\hline
\end{tabular}

empresa A. A análise desta tabela mostra que o peso do ICMS na composição do custo total reduziu em $27 \%$ com o modelo proposto, além do custo de entrega também ter reduzido 3\%. Para que isso ocorresse, houve um acréscimo no custo de transporte de matéria-prima de $20 \%$, como também passou a ter transferências entre unidades, muito embora elas sejam pouco representativas em relação ao custo total. Isso proporcionou uma redução do custo total analisado de $12 \%$, equivalente a $\$ 420$ mil unidades monetárias.

Já a Tabela 14 representa a comparação entre o método empregado pela empresa A e o modelo proposto, considerando apenas os custos adicionais de preparo de espigas nas UBS de GO e SP. Analisando esta tabela, pode-se perceber que o peso do custo adicional de preparo na composição do custo total reduziu em $87 \%$ com o modelo proposto, além do custo de entrega ter sido $1 \%$ menor. Para que isso ocorresse, houve um acréscimo no custo de transporte de matéria-prima de $20 \%$. Isso proporcionou uma redução do custo total analisado de $19 \%$, equivalente a $\$ 450$ mil unidades monetárias. Cabe ressaltar que esta redução foi possível porque havia capacidade disponível na UBS de MG.

Por fim, a Tabela 15 representa a comparação entre o método empregado pela empresa A e o modelo proposto, considerando os custos adicionais de preparo de espigas nas UBS de GO e SP, bem como os de ICMS. Pode-se perceber que o peso do ICMS na composição do custo total reduziu em 30\% com o modelo proposto. Além disso, o peso do custo adicional de preparo na composição do custo total reduziu em $83 \%$ e o de entrega em $6 \%$. Para que isso ocorresse, houve um acréscimo no custo de transporte de matéria-prima de $23 \%$. Isso proporcionou uma redução de $23 \%$ do custo total analisado, equivalente a \$975 mil unidades monetárias. Também neste caso cabe ressaltar que esta redução foi possível porque havia capacidade disponível na UBS de MG.

Estas três comparações mostram que há grandes oportunidades em termos de redução de custos utilizando o modelo proposto, alternativamente à utilização da regra da menor distância. Especificamente no caso da empresa A, cuja estratégia competitiva é voltada à excelência operacional, esta redução de custos implica diretamente
Tabela 14. Comparação entre cenário anterior e o proposto considerando o custo de preparo de espigas.

\begin{tabular}{lccc}
\hline & $\begin{array}{c}\text { Método empresa } \\
(\boldsymbol{\%})\end{array}$ & $\begin{array}{c}\text { c/Prep } \\
(\boldsymbol{\%})\end{array}$ & $\begin{array}{c}\text { Diferença } \\
(\boldsymbol{\%})\end{array}$ \\
\hline Transporte MP & 23 & 34 & 20 \\
Preparo MP & 26 & 4 & -87 \\
Transferência & 0 & 0 & \\
Entrega & 51 & 62 & -1 \\
ICMS & 0 & 0 & \\
Total & 100 & 100 & -19 \\
\hline
\end{tabular}

Tabela 15. Comparação entre cenário anterior e o proposto considerando o ICMS e o custo de preparo de espigas.

\begin{tabular}{lccc}
\hline & $\begin{array}{c}\text { Método empresa } \\
(\boldsymbol{\%})\end{array}$ & $\begin{array}{c}\text { c/ ICMS + } \\
\text { prep (\%) }\end{array}$ & $\begin{array}{c}\text { Diferença } \\
(\boldsymbol{\%})\end{array}$ \\
\hline Transporte MP & 13 & 20 & 23 \\
Preparo MP & 15 & 3 & -83 \\
Transferência & 0 & 2 & \\
Entrega & 28 & 34 & -6 \\
ICMS & 45 & 41 & -30 \\
Total & 100 & 100 & -23 \\
\hline
\end{tabular}

em aumento da competitividade. Comparando-se a vantagem proporcionada exclusivamente pela consideração do ICMS com a do custo adicional de preparo, verifica-se que ambos os ganhos possuem a mesma ordem de grandeza, embora o segundo ganho tenha sido um pouco maior.

Com base nos cenários analisados, foi calculada a utilização das UBS para os recursos em cada cenário. Verificou-se que não houve influência do cenário na utilização das UBS ao longo do tempo. A Tabela 16 mostra a utilização de cada um dos recursos ao longo dos períodos analisados. A análise desta tabela mostra que os recursos mais utilizados são o preparo de espigas, o beneficiamento e a armazenagem de produto acabado, evidenciando que a colheita em grão é pouco realizada e que a armazenagem é feita preferencialmente de produto acabado. Estes recursos também são utilizados para secagem e armazenagem de outras culturas, todavia, isso acaba não sendo limitante, já que a produção de sementes de milho ocupa uma parcela muito pequena da capacidade dos recursos. Além disso, mesmo os recursos mais utilizados possuem uma utilização baixa. No entanto, a agregação da análise de capacidade pode estar mascarando épocas de pico como as que ocorrem na época da colheita das áreas de sequeiro.

Observando-se a utilização por período, o preparo de grãos é utilizado apenas nos três primeiros meses, evidenciando que a colheita em espigas é empregada para os campos de sequeiro, cuja industrialização acontece nos primeiros períodos. Já o preparo de espigas possui uma utilização maior nos três primeiros períodos e no quinto, devido aos picos de demanda analisados na seção 
Tabela 16. Variação da utilização das UBS por período.

\begin{tabular}{lcccccccc}
\hline \multicolumn{1}{c}{ Etapa } & \multicolumn{1}{c}{ Período } \\
\cline { 2 - 8 } & Per1 (\%) & Per2 (\%) & Per3 (\%) & Per4 (\%) & Per5 (\%) & Per6 (\%) & Per7 (\%) & Total (\%) \\
\hline Preparo dos grãos & 7 & 1 & 1 & 0 & 0 & 0 & 0 \\
Preparo das espigas & 47 & 61 & 59 & 20 & 58 & 29 & 26 \\
Beneficiamento & 41 & 29 & 28 & 7 & 21 & 11 & 10 & 24 \\
Arm. dos grãos secos & 2 & 0 & 0 & 0 & 2 & 0 & 0 & 1 \\
Arm. dos prod acabado & 33 & 20 & 20 & 17 & 12 & 7 & 4 & 16 \\
\hline
\end{tabular}

4.1. Comportamento semelhante se observa na etapa de beneficiamento. A armazenagem de grãos secos acontece apenas nos períodos 1 e 5 , quando há sobrecarga no beneficiamento e a demanda não é imediata. Percebe-se que a utilização da armazenagem de produto acabado decresce do primeiro ao último período, representando a produção excedente realizada durante o período de sequeiro.

A Figura 7 mostra a variação da utilização do preparo de grãos de acordo com o cenário: utilizando o método empregado pela empresa, considerando apenas o custo ICMS, considerando apenas o custo adicional de preparo de espigas e considerando o custo ICMS e o adicional de preparo de espigas. Esta figura mostra que nos cenários com a regra utilizada pela empresa e quando se considera apenas os custos de preparo de espigas, a utilização das UBS-GO1 e UBS-GO2 aumenta em detrimento da UBS-MG. Isso ocorre pelo fato da UBS-MG possuir maiores vantagens fiscais que as UBS de GO. Quanto à UBS-SP, ela não prepara grãos no cenário com a regra da empresa, pois a área de colheita em grãos é distante dela, mas se forem consideradas as possibilidades de transferências, bem como as vantagens fiscais, esta unidade passa a ser mais utilizada.

A Figura 8 mostra a variação da utilização do preparo de espigas de acordo com o cenário. Ela mostra que no cenário utilizando a regra da empresa, o preparo de espigas é feito predominantemente na UBS-GO2. No período 2 é utilizada $100 \%$ da capacidade e no período 5, 99\%. Este comportamento ocorre devido às áreas de plantio serem mais próximas a esta UBS. Nos outros cenários, a utilização desta UBS cai pelo menos pela metade. No cenário considerando apenas o custo de ICMS, a utilização das UBS é mais equilibrada: a UBS-MG possui $100 \%$ de utilização no período 3 e a UBS-SP $100 \%$ no período 2 . No entanto, quando passa a ser considerada a redução de custo para o preparo de espigas na UBS-MG, a utilização desta UBS quase dobrou. A UBS-MG tem $100 \%$ de utilização nos períodos 2,3 e 5 .

A Figura 9 mostra a variação da utilização do beneficiamento de acordo com o cenário. Analisando esta figura, percebe-se que com as soluções geradas pelo modelo proposto, a utilização da UBS-GO2 reduz em pelo menos $50 \%$ em detrimento do aumento da utilização da UBS-MG, devido ao custo reduzido de preparo

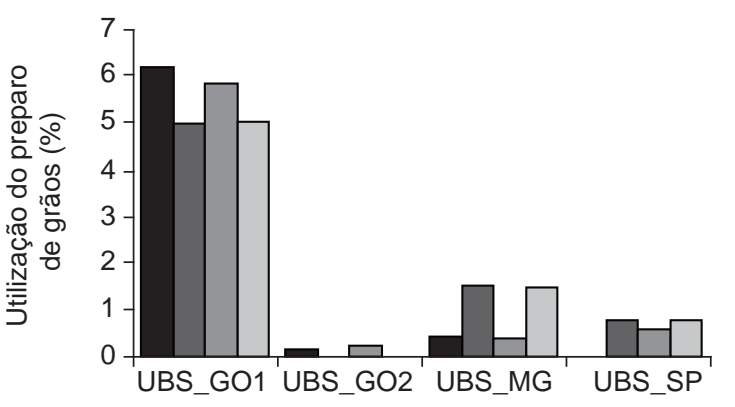

Empresa $\square$ M ICMS $\square$ M prep $\square$ M ICMS + prep

Figura 7. Utilização do preparo de grãos.

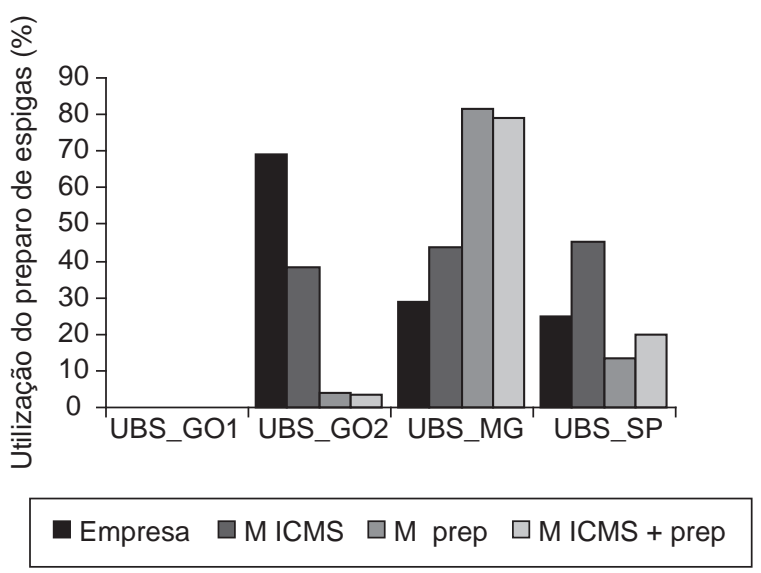

Figura 8. Utilização do preparo de espigas.

de espigas. Nenhuma UBS atingiu utilização de $100 \%$ para qualquer cenário.

\section{Considerações finais}

A produção de sementes de milho envolve uma cadeia de produção agroindustrial complexa, cujos agentes devem primar por oferecer produtos de alta qualidade a um baixo custo para se manterem competitivos no mercado. Neste trabalho analisamos a aplicação de um modelo de programação linear para otimizar o planejamento agregado de produção e logística em um estudo de caso (de uma safra completa) de uma empresa repre- 


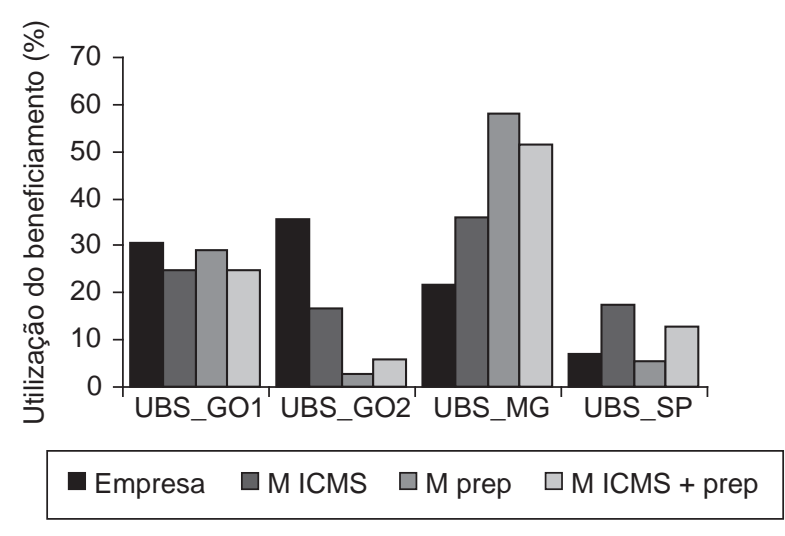

Figura 9. Utilização do beneficiamento.

sentativa do setor de sementes de milho. Os resultados apresentados na seção 4 mostram as vantagens de se utilizar um modelo que considera custos adicionais de preparo de matéria-prima e custos fiscais de ICMS para apoiar decisões de produção e logística. Acreditamos que esta abordagem de planejamento traz vantagens não só para empresas produtoras de sementes de milho focadas em custo, mas também para as que se baseiam em uma estratégia de inovação de produto, conforme discutido na seção 3 .

A análise da utilização das UBS mostrou que há um excesso de capacidade na empresa estudada. Especialmente para o caso de preparo de espigas e beneficiamento, observaram-se grandes variações nos resultados entre os cenários, demonstrando o quanto a abordagem proposta influencia na operação de cada UBS. Além disso, o método empregado pela empresa, que considera apenas a menor distância entre o campo e a UBS, é capaz de gerar soluções factíveis quando existem folgas razoáveis de capacidade na rede. À medida que o nível de utilização dos recursos das UBS cresce e as áreas de plantio passam a ter produção maior do que a capacidade da UBS mais próxima destas áreas, as dificuldades para se encontrar soluções factíveis também aumentam, requerendo uma abordagem mais elaborada de apoio à decisão.

Além disso, apesar das incertezas que existem em relação a quanto e quando ocorrerá a demanda, bem como em quanto e quando produzirá é fundamental que as funções de vendas e produção trabalhem com valores suficientemente próximos de demanda e produção. Isso faz com que se reduzam problemas de informação e comunicação, propiciando a tomada de decisão conjunta e maximizando a eficácia do plano. A abordagem proposta também contribui nesse aspecto, já que o levantamento de dados deve envolver todos os setores da empresa, eliminando por meio de consensos as eventuais ambigüidades e inconsistências nos dados de entrada.

Para o aprimoramento da resposta do plano, é recomendável considerar também os demais custos industriais, considerando outras diferenças entre equipamentos ou instalações, bem como a mão-de-obra. Por fim, a presente abordagem é capaz de gerar soluções para diferentes cenários, demandando relativamente pouco tempo computacional e proporcionando a agilidade necessária na geração e análise destes cenários, permitindo a avaliação dos efeitos de incertezas e potencializando o aprendizado da equipe tomadora de decisão. Uma perspectiva interessante para pesquisa futura é desenvolver outros estudos de caso para avaliar os benefícios da aplicação deste modelo de planejamento otimizado em empresas com estratégias competitivas diferentes da empresa aqui estudada.

\title{
Optimizing production and logistics planning in corn seeds companies: a case study
}

\begin{abstract}
An earlier study (JUNQUEIRA; MORABITO, 2006) presented a linear optimization model to support tactical planning decisions in corn seed production, storage and transportation, aimed at minimizing production, logistics and tax costs and meeting restrictions in harvest scheduling, production capacities and client requirements. The model was tested only in simplified real situations. In the present study, this model was applied to generate an optimized aggregate production plan for a case study of the crop of a corn seed producer during a complete season. Using the model, several scenarios were evaluated considering, for example, different ICMS tax and seed drying costs, and the results obtained were compared with the planning method used by the company. These results indicated that costs in aggregate production and logistic plans can be reduced substantially when the model's premises are taken into account.
\end{abstract}

Keywords: Corn seeds. Aggregate production and logistics planning. Linear optimization. Tax planning. ICMS tax. 
CAIXETA-FILHO, J.V.; SWAAY-NETO, J.M. V.; WAGEMAKER, A. P. Optimization of the Production Planning and Trade of Lily Flowers at Jan de Wit Company. Interfaces, v. 32, n. 1, p. 35-46, 2002.

CARVAlHO, N. M.; NAKAGAWA, J.; Sementes: Ciência, Tecnologia e Produção. 4. ed. Jaboticabal: Funep, 2000, 419 p.

COLIN, E. C.; CIPPARRONE, F. A. M.; SHIMIZU, T. Otimização do custo de transporte na distribuição-armazenagem de açúcar. Produção, v. 9, n. 1, p. 23-30, 1999.

FLEURY, A. C. C.; FLEURY, M. T. L. Estratégias competitivas e competências essenciais: perspectivas para a internacionalização da indústria no Brasil. Gestão \& Produção, v. 10, n. 2, p. 129-144, 2003.

GIORDANO, S. R. Dinamilho: Aquisição de empresa genética vegetal. In: ZYLBERSTAJN, D.(Coord.). Estudos de caso em agribusines: o processo de tomada de decisões nas empresas brasileiras. Porto Alegre: Ortiz, 1993. p. 41-64.

JONES, P.; KEGLER, G.; LOWE, T.; TRAUB, R. Managing the seed-corn supply chain at Syngenta. Interfaces, v. 33, n. 1, p. 80-90, 2003.

JUNQUEIRA, R. A. R. Modelagem do planejamento agrícola, industrial e de transporte de uma empresa de produção de sementes de milho. São Carlos, 2006, 218 p. Dissertação - (Mestrado em Gestão da Produção), Departamento de Engenharia de Produção, Universidade Federal de São Carlos - UFSCAR.

JUNQUEIRA, R. A. R.; MORABITO, R. Um modelo de otimização linear para o planejamento agregado da produção e logística de sementes de milho. Produção, v. 16, n. 3, p. 510-525, 2006.
KAWAMURA, M. S.; RONCONI, D. P.; YOSHIZAKI, H. Optimizing transportation and storage of final products in the sugar and ethanol industry. International Transactions in Operational Research, 2005. (Submetido para publicação).

MUNHOZ, J. R.; MORABITO, R. Um modelo baseado em programação linear e programação de metas para análise de um sistema de produção e distribuição de suco concentrado congelado de laranja. Gestão \& Produção, v. 8, n. 2, p. 139-159, 2001.

PAIVA, R. P. O.; MORABITO, R. Um modelo de otimização para o planejamento agregado da produção em usinas de açúcar e álcool. Gestão \& Produção, v. 14, n. 1, p. 25-41, 2007.

SANTINI, G. A. A Reestruturação da Indústria de Sementes no Brasil: O Novo Ambiente Concorrencial do Segmentos de Milho Híbrido e Soja. São Carlos, 2002, 149 p. Dissertação - (Mestrado em Gestão da Produção), Departamento de Engenharia de Produção, Universidade Federal de São Carlos - UFSCAR.

SLACK, N.; CHAMBERS, S.; JOHNSTON, R. Administração da produção. 2. ed. São Paulo: Atlas, 2002, 754 p.

TAUBE, M. Integrated planning for poultry production at Sadia. Interfaces, v. 26, n.1, p. 38-53, 1996.

YOSHIZAKI, H. Y. Y. Projeto de Redes de Distribuição Física considerando a Influência do Imposto de Circulação de Mercadorias e Serviços. São Paulo, 2002. 144 p. (Tese de Livre Docência em Engenharia de Produção), Escola Politécnica da Universidade de São Paulo - USP.

YOSHIZAKI, H. T. Y.; MUSCAT, A. R. N.; BIAZZI, J. L. Decentralizing ethanol distribution in southeastern Brazil. Interfaces, v. 26, n. 6, p. 24-34, 1996. 


\section{Apêndice 1. Modelo matemático}

Dados de entrada

$\mathrm{Ctr}_{i, j, m, t} \quad$ Custo de frete para transportar uma tonelada do tipo de colheita m do campo i para a UBS j no período $\mathrm{t}$ (em R $\$$ /tonelada).

$C t f_{j, j, t} \quad$ Custo de frete para transportar uma tonelada da UBS j para a UBS jp no período t (em R $\$$ /tonelada).

$C e_{j, k, t}$ Custo de frete para transportar um saco de $20 \mathrm{~kg}$ da UBS j para a região de demanda k no período $\mathrm{t}$ (em R $\$ /$ saco).

$I_{\text {ICMS }} \quad$ Tarifa de ICMS paga pela venda de um saco de $20 \mathrm{~kg}$ da UBS j na região de demanda $\mathrm{k}$.

$P_{h, k} \quad$ Preço de venda do produto h na região de demanda k (em R $\$ / \mathrm{saco}$ ).

$C p_{j, m} \quad$ Custo de preparo da matéria-prima para o tipo de colheita m na UBS j (em R $\$ / \mathrm{saco}$ ).

$C b_{j} \quad$ Custo de beneficiamento na UBS j (em R $\$$ saco).

$\operatorname{EGSecoO}_{h, j} \quad$ Quantidade inicial do híbrido h, armazenada como grãos secos na UBS j (em sacos).

EPFinal0 $_{h, j}$ Quantidade inicial do híbrido h, armazenada como produto final na UBS j (em sacos).

EGSecof $_{h}$ Quantidade final do híbrido h, armazenada como grãos secos (em sacos).

EPFinalf ${ }_{h} \quad$ Quantidade inicial do híbrido h, armazenada como produto final (em sacos).

Dem $_{h, k, t} \quad$ Quantidade demandada do híbrido h, na região mercadológica k e no período $\mathrm{t}$ (em sacos).

PCol $_{h, i, t, t} \quad$ Plano de colheita do híbrido h, com o tipo de colheita m, no campo i e no período t (em sacos).

\% RendPrep $_{m}$ Rendimento no preparo de matéria-prima para o tipo de colheita $\mathrm{m}$.

$\%$ RendBen Rendimento no beneficiamento.

CapPrep $_{j, m} \quad$ Capacidade de preparo da matéria-prima na UBS j do tipo de colheita m (em sacos por dia).

CapSilo $_{j} \quad$ Capacidade de armazenagem em silo na UBS j (em sacos por dia).

CapBen $_{j} \quad$ Capacidade de preparo de beneficiamento na UBS j (em sacos por dia).

CapDep $_{j} \quad$ Capacidade de armazenagem em depósito na UBS j (em sacos por dia).

TurBen $_{j} \quad$ Número de horas de turno de beneficiamento na UBS j (em horas por dia).

Nias $_{t} \quad$ Número de dias no período t (em dias).

Variáveis de decisão

$X_{h, i, j, m, t} \quad$ Quantidade do híbrido h, colhida com o tipo de colheita m no campo i, transportada para a UBS j e nela preparada no período $\mathrm{t}$ (em sacos).

$Y_{h, j, j, t} \quad$ Quantidade do híbrido h, preparada na UBS j e transportada para ser beneficiada na UBS jp no período $\mathrm{t}$ (em sacos).

$Z_{h, j, k, t} \quad$ Quantidade do híbrido h, beneficiada na UBS j e transportada para a região de demanda k no período $\mathrm{t}$ (em sacos).

EGSeco $_{h, j, t} \quad$ Quantidade do híbrido h, armazenada como grãos secos na UBS j e no período t (em sacos).

EPFinal ${ }_{h, j, t}$ Quantidade do híbrido h, armazenada como produto final na UBS j e no período t (em sacos).

Função objetivo

$C T=$ Transporte $M P+$ Preparo $M P+$ Transferência UBS's + Beneficiamento + Entrega

em que:

Transporte MP $=\sum_{h} \sum_{i} \sum_{j} \sum_{m} \sum_{t} \frac{C t r_{i, j, m, t}}{\left(\% \operatorname{RendPrep}_{\mathrm{m}} \% \operatorname{RendBen} * 1000 / 20\right)} \cdot X_{h, i, j, m, t}$

Preparo $M P=\sum_{h} \sum_{i} \sum_{j} \sum_{m} \sum_{t} C p_{j, m} \cdot X_{h, i, j, m, t}$

TransferênciaUBS's $=\sum_{h} \sum_{j} \sum_{j p t} \sum_{t} \frac{C t f_{j, j p, t}}{(\% \operatorname{RendBen} * 1000 / 20)} \cdot Y_{h, j, j p, t}$

Beneficiamento $=\sum_{h} \sum_{j} \sum_{j p} \sum_{t} C b_{j} \cdot Y_{h, j, j p, t}$

Entrega $=\sum_{h} \sum_{j} \sum_{k} \sum_{t} C e_{j, k, t} \cdot Z_{h, j, k, t}+\sum_{h} \sum_{j} \sum_{k} \sum_{t} P_{h, k} \cdot I C M S_{j, k} \cdot Z_{h, j, k, t}$ 
Restrições

$$
\begin{aligned}
& P \operatorname{Pol}_{h, i, m, t}=\sum_{j} X_{h, i, j, m, t} \quad \text { parah }=1 . . H, i=1 . . I, m=1 . . M, t=1 . . T \\
& \sum_{i} \sum_{m} X_{h, i, j, m, t}+\operatorname{EGSeco}_{h, j, t-1}=\sum_{j p} Y_{h, j, j p, t}+\operatorname{EGSeco}_{h, j, t} \\
& \text { para } h=1 . . H, j=1 . . J, t=2 . . T \\
& \sum_{i} \sum_{m} X_{h, i, j, m, 1}+\operatorname{ESSeco0}_{h, j}=\sum_{j p} Y_{h, j, j p, 1}+\operatorname{EGSeco}_{h, j, 1} \\
& \text { parah }=1 . . H, j=1 . . J \\
& \sum_{J p} Y_{h, j, j p, t}+\text { EPFinal }_{h, j, t-1}=\sum_{k} Z_{h, j, k, t}+\text { EPFinal }_{h, j, t} \\
& \text { parah }=1 . . H, j=1 . . J, t=2 . . T \\
& \sum_{J p} Y_{h, j, j p, 1}+\text { EPFinal0 }_{h, j}=\sum_{k} Z_{h, j, k ., 1}+\text { EPFinal }_{h, j, 1} \\
& \text { parah }=1 . . H, j=1 . . J \\
& \sum Z_{h, j, k, t}=\operatorname{Dem}_{h, k, t} \quad \text { parah }=1 . . H, k=1 . . K, t=1 . . T \\
& \sum_{j} \text { EPFinal }_{h, j, T} \geq \text { EPFinalf }_{h} \quad \text { parah }=1 . . H \\
& \sum_{h} \sum_{i} X_{h, i, j, m ., t} \leq \text { Cap Pr } e p_{j, m} . \text { Dias }_{t} \quad \text { para } j=1 . . J, m=1 . . M, t=1 . . T \\
& \sum_{h} \sum_{j p} Y_{h, j, j p, t} \leq \text { CapBen }_{j} . \text { NDias }_{t} \quad \quad \text { para } j=1 . . J, t=1 . . T \\
& \sum_{h} \text { EGSeco }_{h, j, t} \leq \text { CapSilo }_{j} \quad \text { para } j=1 . . J, t=1 . . T \\
& \sum_{h} \text { EPFinal }_{h, j, t} \leq \text { CapDep }_{j} \quad \text { para } j=1 . . J, t=1 . . T \\
& X_{h, i, j, m, t} \geq 0 \quad \operatorname{parah}=1 . . H, i=1 . . I, j=1 . . J, m=1 . . M, t=1 . . T \\
& Y_{h, j, j p, t} \geq 0 \quad \operatorname{parah}=1 . . H, j=1 . . J, j p=1 . . J, t=1 . . T \\
& Z_{h, j, k, t} \geq 0 \quad \text { parah }=1 . . H, j=1 . . J, k=1 . . K, t=1 . . T \\
& E \text { SSeco }_{h, j, t} \geq 0 \quad \operatorname{parah}=1 . . H, j=1 . . J, t=1 . . T \\
& \text { EPFinal }_{h, j, t} \geq 0 \quad \text { parah }=1 . . H, j=1 . . J, t=1 . . T
\end{aligned}
$$

\section{Sobre os autores}

\section{Rogério de Ávila Ribeiro Junqueira}

Logtrac Consultores Associados S/C,

Rua Episcopal, 1675, ap 101, CEP 13560-570, São Carlos, SP, Brasil,

e-mail: rogerio@logtrac.com.br

\section{Reinaldo Morabito}

Departamento de Engenharia de Produção, Universidade Federal de São Carlos,

Caixa Postal 676, CEP 13565-905, São Carlos, SP, Brasil,

e-mail: morabito@ufscar.br

Agradecimentos: Os autores agradecem aos três revisores anônimos pelos úteis comentários e sugestões, e à empresa A pela colaboração durante o desenvolvimento deste estudo. Esta pesquisa contou com o apoio do CNPq e da Logtrac Consultores Associados. 